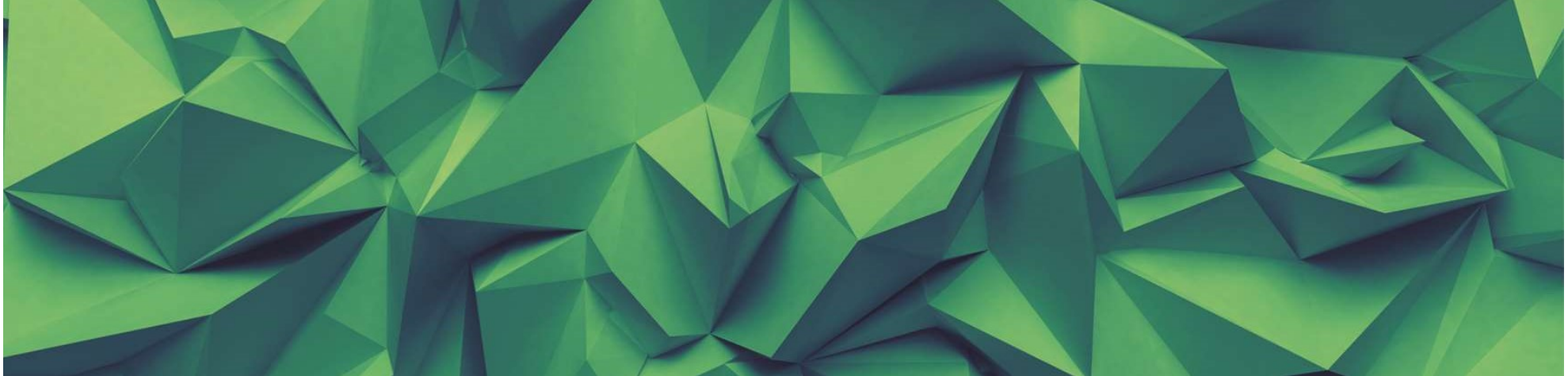

\title{
Sensing Our World Using Wireless Signals
}

\author{
Xiaojiang Chen ${ }^{1,2}$, Jie Xiong ${ }^{3}$, Lili Chen ${ }^{1,2}$, Chao Feng ${ }^{1,2}$, Dingyi Fang ${ }^{1,2 *}$ and Zheng Wang ${ }^{4 *}$ \\ ${ }^{1}$ School of Information Science and Technology, Northwest University \\ 2 Shaanxi International Joint Research Centre for the Battery-free Internet of things \\ ${ }^{3}$ University of Massachusetts, Amherst, USA \\ ${ }^{4}$ Lancaster University, UK \\ * : Corresponding authors
}

In the last few years, pervasive wireless signals have been established as a powerful medium for ubiquitous sensing. Wireless sensing quickly becomes an active cross-disciplinary research area which involves wireless communication, signal processing, human-computer interaction, machine learning and even hardware design. This article provides an accessible introduction to this exciting area and presents the key technologies enabling it. It highlights the latest achievements in this field, and the unaddressed research challenges as well as how emerging wireless standards can better support this promising technique.

\section{INTRODUCTION}

Wireless technologies have achieved a great success in data communication, changing our lives in every aspect in the last two decades. Recent years have also witnessed a surge in Artificial Intelligence, allowing the software to learn automatically from patterns or features in the data by analyzing massive amounts of data with intelligent algorithms. The large amount of data comes from all sources, including sensors deployed around us such as surveillance cameras and wearables worn by us. However, camera-based solutions bring in severe privacy concerns and the elderly are usually reluctant to wear wearables.

Recently, wireless signals such as WiFi, radio-frequency identification (RFID) and sound, have emerged as a powerful medium to sense the human target and also our surrounding environment in a contactless manner. The history of using wireless signals for sensing dates back to the 19th century when people utilized X-rays for imaging ${ }^{1}$ and radar and sonar systems for tracking objects. ${ }^{2}$ Though powerful, these sensing systems employ expensive specialized hardware and need to be operated by professionals, putting them out of the reach of ordinary people.

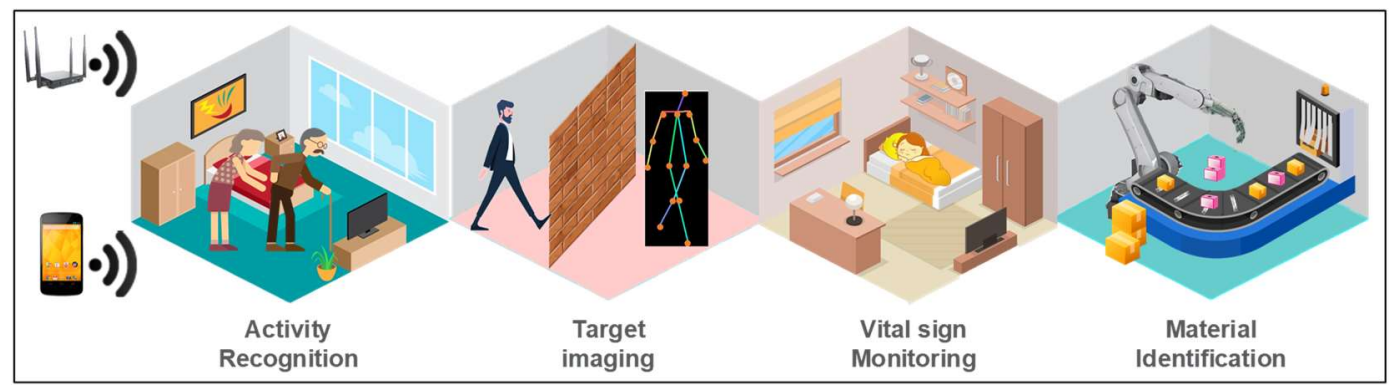

Figure 1. Examples of wireless sensing. 


\section{MAGAZINE NAME HERE}

The latest research exploits wireless signals generated by cheap commercial off-the-shelf (COTS) hardware such as smartphone, WiFi access point and RFID reader for sensing. With the subtle signal variations caused by the target, the target's rich information can be sensed without any device attached to the target. Contactless wireless sensing has enabled a large variety of exciting new applications including indoor localization, 3,4 activity/gesture recognition, ${ }^{5,6}$ fall detection, ${ }^{7}$ respiration monitoring, ${ }^{8}$ emotion sensing, ${ }^{9}$ material identification, ${ }^{10,11}$ room layout mapping, ${ }^{12}$ imaging, ${ }^{13}$ etc. Some of the applications enabled by ubiquitous commodity WiFi hardware are shown in Figure 1. We envision wireless sensing has a tremendous potential to be widely adopted in many of our everyday applications in the Era of Internet of Things (IoT).

As we will show in this article, while there are still outstanding challenges, wireless sensing has moved from a research niche to a mainstream activity. This is, in fact, a dynamic field looking at subjects as diverse as smarthome personalization and fall monitoring to emotion detection and vital sign monitoring. As wireless

sensing has demonstrated convincing promises, it is a natural next step to look at how the emerging wireless standard can better support wireless sensing. In this article we aim to demystify this promising technique, outline the challenges it is facing, discuss how we can better support wireless sensing in standards, and show that this is a trustworthy and exciting research direction.

\section{WORKING MECHANISM OF WIRELESS SENSING}

\section{Working Example}

To illustrate how wireless sensing works, we take gait identification depicted in Figure 3(a) as one example. The sensing task is to identify which user has walked pass the scene by identifying his unique gait pattern.

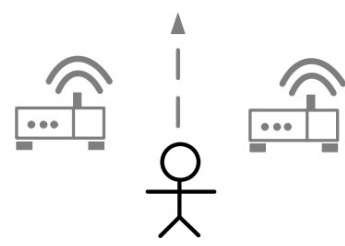

(a)

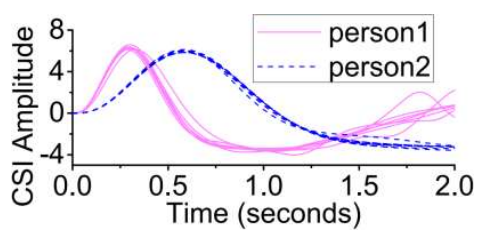

(b)

Figure 2. The working mechanism of wireless sensing. (a) shows a typical set up where two wireless devices are deployed to collect the CSI values when a user walks pass the devices. (b) shows the measured CSI for two individuals, where the resulted CSI amplitudes are relatively consistent for the same person across multiple walks but are sufficiently different for different people.

Knowing this information allows one to -- for example -- personalize the light setting of a smart home. Here, two wireless devices (a sender and a receiver) are used to measure how the user's movement affects the wireless channel metric, such as the channel state information (CSI) or received signal strength indicator (RSSI). The wireless signals -- called "multipath" -- will bounce off the wall, furniture, and human body. The human movement or activity can lead to a change on the amplitude and phase of the signal reflected from the human target. By measuring how the wireless signal is uniquely affected by the activity and comparing the measurement against pre-collected training data, one can infer what activity has been performed and by whom. Figure 2(b) shows the measured CSI amplitudes of two users in our scenario. In this case, each user walks through our scene five times and the CSI amplitudes for the 10 walks are shown. Figure 2(b) suggests that CSI can be used for user identification, because the CSI measurements for the same person are relatively consistent and are sufficiently different between the two users.

Note that although wireless technology and the sensing method would vary depending on the sensing task, this example demonstrates the basic working principle of wireless sensing. 


\section{Overall Framework of Wireless Sensing}

A wireless sensing framework typically comprises three components for collecting, transmitting and processing sensing data. Such a framework could be implemented by utilizing existing IoT infrastructures, to leverage the pervasive computing devices.

The first step of wireless sensing is to gather the sensing data, e.g., wireless signal channel metrics, through computing devices such as wireless routers or RFID readers. The collected data needs to be stored in a specific format. One of the challenges for data collection is that the volume of collected sensing data is often large. Processing such large volume of data would require computation power that is often beyond the capability of the data collection device. As a result, the collected data often needs to be transmitted to be processed by a more powerful computing device, such as a high-performance PC. After transmitting the data to the processing device, the data will then be analyzed and processed to extract useful information to e.g., identify the target activities. We note that each of these three steps pose unique technical challenges to the current computing infrastructures and network protocols, and we provide a discussion on these issues later in the article.

\section{Choices of Wireless Sensing Signals}

As summarized in Table 1, a wide range of wireless signals have been employed in prior work for information sensing. These include WiFi, RF, acoustic, LoRa and visible lights, $60 \mathrm{GHz}$ and ultra-wideband (UWB). Signals like WiFi, acoustic, and visible lights are readily available on commercial off-the-self devices (such as smartphones and smart light bulbs), while others like UWB and $60 \mathrm{GHz}$ often require dedicated hardware to operate. As can be seen from the table, this technique has been demonstrated to be effective on a wide range of standards.

Table I. Consumer-grade wireless signals used in prior sensing tasks

\begin{tabular}{|c|c|c|c|c|c|c|c|}
\hline Properties & WiFi & RFID & UWB & Acousti-c & LoRa & $60 G H z$ & $\begin{array}{c}\text { Visible } \\
\text { Light }\end{array}$ \\
\hline $\begin{array}{l}\text { Communic- } \\
\text { ation Range }\end{array}$ & $35 \mathrm{~m}$ & $10 \mathrm{~m}$ & $10-20 m$ & $2-3 m$ & $15 \mathrm{~km}$ & $10 \mathrm{~m}$ & $1.4 \mathrm{~km}$ \\
\hline Frequency & $\begin{array}{c}2.4 / 5 \mathrm{GH} \\
z\end{array}$ & $\begin{array}{c}902.75- \\
927.25 \mathrm{MHz}\end{array}$ & $\begin{array}{c}3.1- \\
10.6 \mathrm{GHz}\end{array}$ & $17-24 \mathrm{KHz}$ & $\begin{array}{l}\text { 868/903- } \\
927 \mathrm{MHz}\end{array}$ & $57-64 \mathrm{GHz}$ & $\begin{array}{c}380- \\
790 \mathrm{THz}\end{array}$ \\
\hline Bandwidth & $\begin{array}{c}20 / 40 \mathrm{M} \\
\mathrm{Hz}\end{array}$ & $24.5 \mathrm{MHz}$ & $1 \mathrm{GHz}$ & --- & $\begin{array}{c}125 / 250 / 50 \\
0 \mathrm{KHz}\end{array}$ & $7 \mathrm{GHz}$ & --- \\
\hline Metrics & CSI, RSSI & $\begin{array}{c}\text { Phase, RSSI, } \\
\text { Doppler }\end{array}$ & $\begin{array}{c}\text { Phase, } \\
\text { RSSI }\end{array}$ & $\begin{array}{c}\text { Phase, } \\
\text { RSSI }\end{array}$ & $\begin{array}{l}\text { Phase, RSSI, } \\
\text { Frequency }\end{array}$ & RSSI & RSSI \\
\hline Cost* & $\$ 19.9$ & $\$ 0.239$ & $\$ 555$ & $\$ 99.99$ & $\$ 30$ & $\$ 43,822$ & $\$ 30.09$ \\
\hline
\end{tabular}

*Prices were collected from amazon.com as of January 2019 for standard components.

Which signal is best for a particular sensing task, is the $\$ 64,000$ question. The is a difficult question and the answer is, it depends. For example, while WiFi is ubiquitous indoors, it is limited in the communication range $(15-50 \mathrm{~m})$ and consumes a significant amount of power. In contrast, LoRa supports a much longer range up to $15 \mathrm{~km}$ and needs much less power to operate, but it has a much smaller bandwidth and is more sensitive to multipath and interference. On the other hand, acoustic signals can achieve a surprisingly high sensing accuracy at millimeter level ${ }^{14}$ due to the much smaller propagation speed in the air. However, the sensing range of acoustic signals is usually below $1 \mathrm{~m}$. It is thus important to note that there is no "one-size-fits-all" sensing signal, and the choice depends on the trade-off between the precision requirements, the sensing range, and the budget. 


\section{WIRELESS SENSING APPLICATIONS}

Wireless sensing has been demonstrated to be effective on a wide range of tasks from localization to activity recognition and material identification.

\section{Localization and Tracking}

Target localization and tracking are two popular sensing applications. WiFi and RFID are the popular technologies used for this task, due to the low-cost and wide-scale deployment of the hardware. Acoustic signals are usually employed for high-accuracy tracking in a small range.

Broadly speaking, the localization and tracking technologies can be divided into two categories: 1) device based and 2) device-free based. The general idea of a device-based approach is to measure the signal that transmitted from the user-end device to the remote receivers, and then determine the user location based on the angle of arrival (AoA), time of flight (ToF), signal amplitude, etc. By contrast, a device-free approach does not require the user to carry a specific device. It works by relying on measuring how the user affect the ambient wireless signals to determine the user's location or moving pattern.

While the amplitude-based approach is simple and cost efficient, it suffers from poor localization accuracy (especially in non-line-of-sight conditions) due to additional signal attenuation resulting from transmission through walls and other big obstacles and severe amplitude fluctuation due to multipath fading and indoor noise. The accuracy of the AoA based methods depends on the size of the antenna array at the receiver while the frequency bandwidth is the key determining factor for ToF based methods. Obviously, the frequency range is restricted by different industrial standards. For example, techniques built upon the 802.11 standard family can be used the $2.4 \mathrm{GHz}$ or $5 \mathrm{GHz}$ frequency bands. UWB with a wide frequency band is thus an ideal candidate for ToF-based localization and tracking. Most of the device-free based localization approaches require constructing fingerprint, by first collecting the wireless fingerprints (e.g., RSSI or CSI) at chosen locations. During deployment, it compares the measured CSI/RSSI readings against the pre-collected fingerprints to infer the location of the target. Such an approach is easy to be deployed but requires high human-labor cost and its accuracy is susceptible to the environmental changes such as furniture movement.

\section{Activity and Behavior Recognition}

Human activity and behavior recognition is another area where wireless sensing has demonstrated great potential.

Some of the early work in the area use multiple transmitters and receivers to construct a 3D lattice of wireless links to identify if there are human movements. The latest efforts use a single device equipped with multiple antennas to realize activity recognition. For examples, WiSee ${ }^{15}$ is able to differentiate 9 commonly seen body gestures with the help of machine learning methods. A more recent work illustrates the possibility of using wireless signals for measuring human's breathing and heart rate. ${ }^{8}$ The idea is that the chest movement caused by breathing would change the signal reflections, which in turn allows one to capture the breathing event and count the heart rate at high enough accuracies. By precisely counting the heart beats, one can even predict a user's emotion. $^{9}$

Machine learning based classifiers have been widely used in activity recognition. The effectiveness of these methods in general depends on the quality of the training data and features for capturing the characteristics of the activity. As a result, some of the recent research efforts turn into seeking ways to automatically identify useful features ${ }^{16}$ or reduce the human involvements (and thus the labor cost) for generating high-quality training data. ${ }^{13}$

\section{Target Imaging and 3D Reconstruction}

Target imaging is another area that has attracted extensive attentions. Traditional imaging systems like Xrays and CT employ dedicated hardware with high frequency, large bandwidth and antenna arrays, which are extremely expensive and usually large in size. In recent years, researchers attempt to employ RF signals to image a target's shape. For instances, the 802.11 standard-based WiFi system Wision ${ }^{17}$ utilizes the reflected 
signals from the target for imaging. Its imaging performance is quite coarse due to the small bandwidth of commodity WiFi. Tagscan ${ }^{10}$ leverages the RFID signal change at each angle to obtain the target width information and stitches together the width information at many angles to obtain the horizontal cut image of the target.

Meanwhile, more advanced methods by using frequency-modulated continuous-wave (FMCW) technology for imaging have been proposed. RF-Capture ${ }^{18}$ can reconstruct the coarse skeleton of a human target behind a wall by extracting the signal reflections from body parts of a moving target. The latest work RF-Pose $3 \mathrm{D}^{13}$ infers $3 \mathrm{D}$ human skeleton by incorporating RF signal (FMCW) and visual stream. It first leverages deep CNN to infer the person's 3D skeleton, and then uses the visual stream to label different target skeletons. RF-Pose3D can track each key point on the human body at a centimeter-level accuracy.

\section{Object Material Identification}

Material identification is another exciting direction for wireless sensing. It can be adopted in applications such as detecting concealed weapons and expired milk.

Existing material identification systems such as X-rays, CT, MRI and B-scan ultrasonography employ extreme high frequency signals for sensing. They are expensive and need professionals to operate. Some of the recent work utilize the low-cost wireless signals to sense the target material. TagScan ${ }^{10}$ identifies the target material by detecting the changes of the RSSI and phase when the RFID signal penetrates through the target and it is sensitive enough to differentiate between Coke and Pepsi. Wang et al. ${ }^{19}$ explores the feasibility of using CSI from COTS WiFi to detect suspicious objects (i.e., liquid objects and metal) hidden in baggage. LiquID ${ }^{11}$ employs the UWB signals to identify liquids at high accuracies.

\section{Vibration Detection}

Vibration detection plays a vital role in many applications, such as malfunction detection in electronic instrument and monitoring the displacement in vehicle. Traditional vibration sensing schemes ${ }^{20}$ require dedicated sensors, which are very expensive. Furthermore, most of them have a limit monitoring area.

To avoid these drawbacks, TagBeat ${ }^{21}$ proposes to utilize commodity RFID to achieve sub-millisecond vibration sensing. In order to magnify tiny vibration signal induced by micro-vibration, TagBeat exploits a special signal processing technique to virtually extend the vibration radius, which is equivalent to multiplying vibration amplitude by a constant. Note that the side-effect of magnification is that noise is amplified too. In order to avoid data collision, Gen2 utilizes the Aloha type MAC algorithm, in order to restrict the reader data rates to a certain range. TagBeat also proposes a compressive sensing model to reconstruct the vibration signal to overcome the challenge of detecting high-frequency vibration with a limited sampling rate.

\section{CHALLENGES OF WIRELESS SENSING}

This article has by and large been very upbeat about wireless sensing. However, there are a number of hurdles to overcome before we can make wireless sensing a practical reality.

Multipath effect: As wireless signals can be reflected, diffracted and bounced off surrounding objects like walls and furniture in an indoor environment, multiple copies of the same signal may reach the receiver with different phase rotations and power attenuations. This phenomenon is known as the multipath effect. Multipath makes it difficult to obtain clear signal information (RSSI, phase, AoA, ToF, etc.) from each single signal path which is required for accurate sensing. Recent literature has proposed to select those sub-carriers that are not affected greatly by multipath for sensing ${ }^{22}$ while totally removing the effect of multipath remains an open problem. There are other schemes for dealing with the multipath effect, but they often require a large bandwidth or a large-size antenna array.

Many wireless sensing methods work by observing at the receiver side, how the signals are affected by the target object. The reflected/diffracted signals are much weaker than the direct-path signal and are usually weaker compared to those bounced from surrounding large-size non-target objects (e.g., walls or furniture), making it non-trivial to employ the reflected/diffracted signals for sensing. Interference from other people make 
it more challenging to have wireless sensing working in reality. Therefore, dealing with multipath and interference is critical in bridging the gap between sensing in the lab and sensing in reality.

Clock synchronization: Wireless sensing systems often employ one transceiver pair to track objects and activities. To obtain accurate ToF information, tight clock synchronization between the transceiver pair is essential because the time error of ten nanoseconds can translate to a localization error of $300 \mathrm{~cm}$. However, obtaining tight time synchronization wirelessly is still very challenging. One commonly seen solution is to use a wire to synchronize the clocks between the transceiver pair which however, greatly limits the deployment flexibility of the system.

Deployment: The performance of a wireless sensing system highly depends on how the transmitter(s) and receiver(s) are deployed in the environment. Determining the optimal device deployment can be challenging due to the complex multipath situation and the mobility of the target. ${ }^{6}$ The optimal deployment strategy also changes if the environment gets changed such as adding or removing some furniture. Such a change often requires recollecting the training data and updating the sensing model, which is often associated with human involvement at a high cost. Recent work tries to address the issue with machine learning methods to generate synthetic training examples. ${ }^{16}$ While learning-based methods are able to reduce the cost for collecting training data, they cannot eliminate it. Even for those model-based methods without learning, ${ }^{6}$ the transceiver pair's locations may need to be adjusted when the target moves.

Evaluation: Currently, a proposed sensing method is typically evaluated on bespoke datasets and the experiments are conducted in specific environments and settings. Lacking common datasets make it difficult to fairly compare the performance of different approaches. Many existing approaches built upon machine learning methods work like a blackbox without revealing the deep insight why they work. The black-boxing mechanism prevents the quality of the model from being questioned unlike analytical methods. As a result, reviewers now have to scrutinize that the experiments were fairly done. This means all the implementation and test data must be publicly available for scrutiny. We notice that some of the conferences are now promoting reproducibility by encouraging the authors to open-source their code and share the datasets. ${ }^{23} \mathrm{We}$ hope these activities can result in more widely accepted public datasets and evaluation standards.

\section{MAKING SUPPORT OF WIRELESS SENSING A STANDARD}

Wireless sensing with cheap commodity hardware has demonstrated its feasibility to sense the human body and our environment in a revolutionary contactless manner. We envision this to be just the promising beginning and many new exciting applications are coming including in-body localization, through-wall imaging, sensing of high-speed mobile objects (driverless vehicles, UAV), etc. Today, sensing is still a side product of wireless data communication.

While RSSI information is widely available, the CSI is not easily accessible at most commodity hardware and this becomes a major barrier for ubiquitous sensing. We believe this situation may get changed with more compelling and even killer applications enabled by sensing in the future and the major wireless hardware companies may release more physical layer signal information to support wireless sensing.

An interesting question to ask is: "how we can make the support of wireless sensing as part of the standard?" To improve the practicability of wireless sensing, many recent works have tried to exploit the COTS hardware that follows the industrial standards including the EPC standard for RFID and 802.11 for WiFi sensing. While many recent wireless sensing solutions try to utilize COTS hardware. The lack of support in the wireless standards make it difficult to obtain some crucial information such as CSI. Often, researchers have to hack into the low-level hardware driver to obtain such information, despite that it is readily available at the physical layer. As a result, the developed solution only works on a certain type of devices where a workaround solution is available. It is no doubt that the lack of standard support has become a major barrier for the adoption of wireless sensing. If providing information like CSI can become an industry standard or be part of emerging wireless protocol - just like providing the wireless signal strength metric is now become a standard function of any wireless router-- it would certainly help this promising technique to be taken at a larger scale.

It is currently challenging to effectively communicate the low-level wireless information from the physical layer all the way to the application layer. This is partly because the network stack is not designed for wireless 
sensing. For example, currently to obtain CSI would require hacking into the physical layer protocol and heavy developer efforts to make sure the low-level information can be interpreted and processed by the application. This cross-layer communication and data conversion requires significant computation power which often is beyond the capability of the end-user's device (such as an embedded wireless router). If we can redesign the interfaces among network layers to allow easy data collection, transmission and processing, we can then allow wireless sensing to be taken at a larger scale. For example, one can revise the physical layer design to enable fast path searching in the data-link layer, which represents a significant cost of the current wireless sensing solutions. Such an effort will also require redesigning the network protocol, to standardize the data format to provide a common working language across network layers and computing devices work at different layer.

Many wireless sensing applications like gait identification or gesture recognition requires monitoring how the wireless signal is affected by human activities. Such applications would raise privacy and data security concerns. While data encryption permits secure communication for data transmission, it does not eliminate the privacy concern, especially when the data are stored and processed on a remote computing server. Fortunately, some of the recent developments on differential privacy ${ }^{24}$ have demonstrated great potential on preserving data privacy. It would be interesting to see how these techniques can be applied to wireless sensing applications.

\section{CONCLUSION}

Wireless sensing is an exciting cross-disciplinary area touching wireless communication, signal processing, machine learning, ubiquitous computing and HCI. This article briefly introduces the current state of wireless sensing with commodity hardware. We try to deliver a general picture of this area and present the research topics, challenges and future trends. We believe wireless sensing is still in its early stage and there are a lot of opportunities and challenges ahead, calling for creative and high-impact research to be conducted in this exciting area.

\section{ACKNOWLEDGEMENTS}

We thank the anonymous reviewers for their valuable comments and helpful suggestions. This paper is supported by the National Natural Science Foundation of China under grant agreements 61772422 , 61672428, 61772422 and 61872294; the Science and Technology Innovation Team Support Program of Shaanxi Province, China (2018TD-O26); the UK Engineering and Physical Sciences Research Council (EPSRC) through grant agreements EP/M01567X/1 (SANDeRs) and EP/M015793/1 (DIVIDEND); and the Royal Society International Collaboration Grant (IE161012).

\section{References}

1. A. G. Filler, "The history, development and impact of computed imaging in neurological diagnosis and neurosurgery: Ct, mri, and dti," Internet Journal of Neurosurgery, 2010 L. Y et al., "Tagoram: real-time tracking of mobile rfid tags to high precision using cots devices," in MobiCom, 2014.

2. W. W. L. Au and D. W. Martin, "Sonar discrimination of metallic plates by dolphins and humans," 1988.

3. J. Xiong and K. Jamieson, "Arraytrack: a fine-grained indoor location system," in NSDI, 2013.

4. L. Yang et al., "Tagoram: real-time tracking of mobile rfid tags to high precision using cots devices," in MobiCom, 2014

5. W. Wang et al., "Understanding and modeling of wifi signal based human activity recognition," in MobiCom, 2015.

6. H. Wang et al., "Human respiration detection with commodity wifi devices: do user location and body orientation matter?" in UbiComp, 2016.

7. Y. Wang et al., "Wifall: Device-free fall detection by wireless networks," IEEE Transactions on Mobile Computing, 2017.

8. F. Adib et al., "Smart homes that monitor breathing and heart rate," in CHI, 2015.

9. M. Zhao et al., "Emotion recognition using wireless signals," in MobiCom, 2017.

10. J. Wang et al., "Tagscan: Simultaneous target imaging and material identification with commodity rfid devices," in MobiCom, 2017

11. A. Dhekne et al., "Liquid: A wireless liquid identifier," in MobiSys, 2018

12. C. X. Lu et al., "Simultaneous localization and mapping with power network electromagnetic field," in MobiCom, 2018.

13. M. Zhao et al., "Rf-based 3d skeletons," in SIGCOMM, 2018. 
14. W. Wang et al., "Device-free gesture tracking using acoustic signals," in MobiCom, 2016.

15. Q. Pu et al., "Whole-home gesture recognition using wireless signals," in MobiCom, 2013

16. J. Zhang et al., "Crosssense: Towards cross-site and large-scale wifi sensing," in MobiCom, 2018.

17. D. Huang et al., "Feasibility and limits of wi-fi imaging," in SenSys, 2014.

18. F. Adib et al., "Capturing the human figure through a wall," ACM Transactions on Graphics, 2017

19. C. Wang et al., "Towards in-baggage suspicious object detection using commodity wifi," in CNS, 2018.

20. "Lion precision," in http://www.lionprecision.com/.

21. L. Yang et al., "Making sense of mechanical vibration period with submillisecond accuracy using backscatter signals," in MobiCom, 2016.

22. J. Wang et al., "Lifs: Low human-effort, device-free localization with fine-grained subcarrier information," in MobiCom, 2016.

23. Online resource (URL): https://www.artifact-eval.org/.

24. Z. Qin et al. "Heavy Hitter Estimation over Set-Valued Data with Local Differential Privacy," in CCS, 2016. 